\title{
TRIAD HISTOGRAM TO ENHANCE CHEST X-RAY IMAGE
}

\author{
R.Senthilkumar ${ }^{1}$, Dr. M. Senthilmurugan ${ }^{2}$ \\ Research Scholar, Research \& Development Centre, Bharathiyar University,Coimbatore, India ${ }^{1}$ \\ Professor, Dept. of Computer Applications, Bharathiar College of Engg., \& Technology, Karaikal, Pondicherry (U.T)
}

\begin{abstract}
The objective of Digital Image processing(DIP) refers to manipulate and enhance the image quality. There are plenty of image processing applications and problems are there, namely image representation enhancement, restoration and compression etc., In this paper, only three types of histogram modification methods (i.e. HE, AHE, \& CLAHE) are discussed and implemented in MATLAB. The performance of these techniques is then compared using various parameters such as Peak signal to noise ratio (PSNR), Mean squared error (MSE), Signal to noise ratio (SNR), Absolute mean brightness error (AMBE) and Entropy. The results shows that CLAHE is efficient when compared to other two methods.
\end{abstract}

Keywords: DIP, Representation, Restoration, Compression, Medical Image Enhancement, Histogram.

\section{INTRODUCTION}

Whenever an image is converted from one shape to for enhancing x-ray images. Jagatheeswari1 et al.[3] used another some form of dilapidation occurs at the output. To a median filter ( $3 \square 3$ mask) with histogram equalization. improve such dilapidation, the application of restoration To avoid impulse noise the enhanced image has been and/or enhancement can be used[1]. Restoration requires passed through a median filter. Sarage and Sagar an a priori model of the degradation process. When no Jambhorkar [4] had proposed filtering techniques to such knowledge is available the quality of an image may enhance the contrast of x-ray images which were distorted be improved for specific application by some adhoc due to noise and blurring. This technique involved the use process called image enhancement. The main purpose of of different filters such as median filter to remove noise digital image processing(DIP) method is to get better and mean filter to remove the high frequency illustrative information for human elucidation. Moreover, details.Tiwari and Yardi [5] proposed an adaptive the enhancement process does not augment the inborn technique to improve the contrast quality of dental X-ray information content in the data. But it does increase the image using the Laplacian-of-a-Gaussian (LoG) filter. active range of the selected features. So that they can be They had proposed to replace the fixed power detected easily. Image enhancement includes graylevel transformation function from converting original image and contrast manipulation, noise reduction, edge into a mid-range intensity image. Ritika [6] has proposed a crispening and sharpening, filtering and so on. The technique to enhance the contrast of the medical images greatest predicament in image enhancement is quantifying using mathematical morphology with the help of the standard for fortification. Filtering is one of the multiscale structuring element. However, there was a augmentation techniques which is used to get rid of extra slight amplification of noise in this method. Kalyan noise from the picture. It is also used for image Chatterjee et al [7] had implemented neuro-fuzzy sharpening and smoothening. Mean intensity of the image inference system to obtain the clear image. Contrast may loss and computational time is high while enhance an enhancement was performed using Histogram image using local augmentation technique.These equalization. Image enhancement using histogram boundaries can be overcome by contrast enhancement. equalisation was best suited for medical images.

Not only this task, but also to make the image brighter, visual and detail worth full. Therefore a large number of image enhancement techniques are experimental and necessitate interactive procedures to obtain satisfactory results. However, image enhancement remains a very important topic because of its usefulness in all image processing applications. The remainder of this paper is organized as follows : In section 2 survey on existing works, section 3 presents the materials and methods. Results and discussions in section 4 . In section 5 conclusion to this paper is presented.

\section{RELATED WORKS}

Frosio and Borghese [2] have presented that the unsharp mask technique is one of the common UTILIZED METHODS

Siti Arpah Ahmed et al [8] proposed an algorithm to analyse the image enhancement technique for dental X-ray image interpolation and compared four enhancement techniques namely,AHE,CLAHE,MAHE and SCLAHE. They concluded that the Adaptive Histogram Equalisation enhanced the image with better contrast. Mohammed et al [9] proposed spatial enhancement and power law transformation for enhancing medical images. It was concluded that as the power law increases, the brightness of the image increases. However, further enhancement can be made using other enhancement techniques

\section{III.MATERIALS AND METHODS}

The contrast stretching process plays an important role in enhancing the quality and contrast of medical images. 
Medical images are usually fused, subject to high inconsistency and composed of different minor structure. In medicine all the data and related health information are stored as visual information in the form of $\mathrm{x}$-rays, ultrasound or other scanned images for diagnosis and monitoring purposes. For medical images, especially x-ray chest images, texture feature extraction is a more difficult task as well as the $\mathrm{x}$-ray images are gray-level images with poor contrast, high noise and important information often exist in a relatively small local area of the image, which are quite different from regular images. Regular texture extraction methods are not very suitable for x-ray images. The goal of medical information systems have often been defined as the delivery of the needed information at the right time, at the right place in order to improve the quality and efficiency of care process. If the original image is of rather low-contrast and does not contain much information stretching the contrast can only accomplish so much[10]. To enhance the contrast of chest $\mathrm{X}$-ray image which is used in this study the techniques HEQ,AHEQ,CLAHEQ are used.

\section{A Histogram Processing}

Histogram processing is used in image enhancement. The information inherent in histogram can also used in other image processing application such as image segmentation and image compression. A histogram simply plots the frequency at which each grey-level occurs from 0 (black) to 255 (white). The histogram is a discrete function that is shown in figure. Histogram represents the frequency of occurrence of all gray-level in the image, that means it tell us how the values of individual pixel in an image are distributed. Histogram is given as :

$$
\mathrm{h}(\mathrm{rk})=\mathrm{nk} / \mathrm{N}
$$

Where rk and nk are intensity level and number of pixels in image with intensity respectively. The contrast can be improved by scaling the graylevel of each pixel,so that image graylevel occupy entire dynamic range available. The operation may be called "histogram stretching." Fig.1

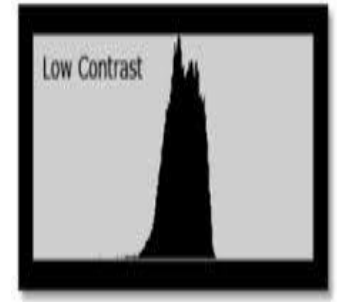

(a)

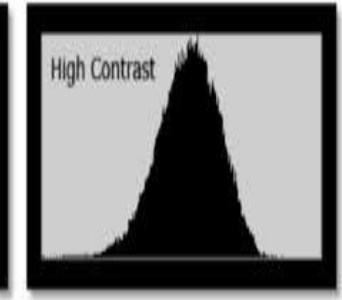

(b)

\section{B Histogram Equalization :}

Histogram equalization is the most well-liked algorithm for contrast enhancement due to its efficacy and simplicity. It can be classified into two branches according to the transformation function used: global or local. Global histogram equalization is simple and fast, but its contrast- enhancement power is relatively low. Local histogram equalization, on the other hand, can enhance overall contrast more effectively, but the complexity of computation required is very high due to its fully overlapped sub-blocks Global histogram equalization method is simple and powerful, but it cannot adapt to local brightness features of the input image because it uses only global histogram information over the whole image. This fact limits the contrast-stretching ratio in some parts of the image, and causes significant contrast losses in the background and other small regions. To overcome this limitation, a local histogram-equalization method has been developed, which can also be termed block-overlapped histogram equalization.

\section{Adaptive Histogram Equalization (AHE)}

The standard histogram equalization is effective and simple method for contrast enhancement but for medical images most of the time it produces excessive contrast enhancement due to lack of control for the level of enhancement. Adaptive histogram equalization (AHE) is a computer image processing technique used to improve contrast in images. Moreover, the Histogram equalization amplifies the image noise and increases visual graininess or patchiness. To overcome these drawbacks, many variants of $\mathrm{HE}$ have been proposed. In medical imaging such as mammogram image enhancement local contrast are more important than global contrast. In such type of applications Global Histogram Equalization (GHE) is insufficient because it cannot deal with local features of original image due to its global nature. Adaptive Histogram Equalization (AHE) method will perform throughout all pixels in the entire image and maps gray level using local histograms, but it takes more time. The basic idea behind the scheme is to divide the image into a grid of rectangular contextual regions, and to apply a standard histogram equalization in each AHE is able to overcome the limitations of the standard equalization method and achieves a better presentation of information present in the image

\section{Contrast Limited Adaptive Histogram Equalization (CLAHE)}

A more advanced version of histogram equalization, adaptive histogram equalization, makes the assumption that the image varies significantly over its spatial extent.

CLAHE was originally developed for medical imaging and has proven to be successful for enhancement of lowcontrast images such as x-ray images and portal films CLAHE, is an improved version of Adaptive Histogram Equalization (AHE).Both overcome the limitations of standard histogram equalization. The algorithm divides the image into smaller tiles, applies histogram equalization to each tile, then interpolates the results. MATLAB's implementation[11], adapthisteq(), includes limits on how much the contrast is allowed to be changed, called contrast-limited adaptive histogram equalization, or 
CLAHE for short. Again, CLAHE will modify the image • in strange ways, but those may be better for certain tasks. Each tile's contrast is enhanced, so that the histogram of the output region approximately matches the histogram specified by the 'Distribution' parameter. The neighboring tiles are then combined using bilinear interpolation to eliminate artificially induced boundaries. The contrast, especially in homogeneous areas, can be limited to avoid amplifying any noise that might be present in the image.

To measure the image quality, various metrics are used in this study :

SNR, is defined as the ratio of signal power to the noise power, often expressed in decibels. Higher the SNR value betters the reconstructed image. Which is calculated by

$\mathrm{SNR}=10 \log 10\left(\frac{\text { Psignal }}{\text { Pnoise }}\right)=$ Psignal, $\mathrm{dB}-$ Pnoise $\mathrm{dB}$

PSNR, is the estimation standard of the reconstructed image quality. It is measured in the decibels $(\mathrm{dB})$ and it is given by

$$
\text { PSNR }(\mathrm{dB})=10 \log _{10}(255 \mathrm{X} 255) / \mathrm{MSE}
$$

Where the value 255 is the maximum possible value that can be attained by the image signal. Mean square error is defined as where $\mathrm{M}^{*} \mathrm{~N}$ is the size of the original image. Higher the PSNR value betters the reconstructed image. MSE, is the average squared difference between the reference signal and distorted signal. It is given by

MSE $=\frac{1}{m x n} \sum_{i=0}^{m-1} \sum_{i=0}^{i-1}[\mathrm{I}(\mathrm{i}, \mathrm{j})-\mathrm{K}(\mathrm{i}, \mathrm{j})]^{2}$

Entropy, is a useful tool to measure the richness of the details in the output image. It is given by

$\operatorname{Ent}[\mathrm{p}]=-\Sigma \mathrm{k}=0(\mathrm{k}) \log 2 \mathrm{p}(\mathrm{k})$

AMBE, is the difference between the brightness of the original image and enhanced image. It is given by

$$
\mathrm{AMBE}=|\mathrm{E}(\mathrm{x})-\mathrm{E}(\mathrm{y})|
$$

Where $\mathrm{E}(\mathrm{x})$ is the average intensity of the input image and $\mathrm{E}(\mathrm{y})$ is the average intensity of enhanced image. The value of AMBE should be as little as possible.

Procedure to enhance the input image :

- $\quad$ Download the chest X-ray image .

- $\quad$ save the image under .jpg extension

- $\quad$ apply the proposed methods (ie HE,AHE

\& CLAHE) generate the output images along with

scatter diagram.

- measure the image quality, using the metrics.

\section{RESULTS \& DISCUSSIONS}

The three image enhancement techniques such as HE, AHE and ,CLAHE are implemented using MATLAB. The performance of all these image enhancement techniques are analyzed for chest X-ray image are presented.

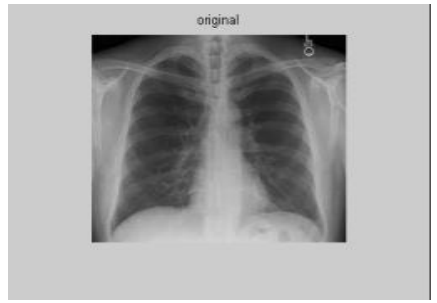

(a)original

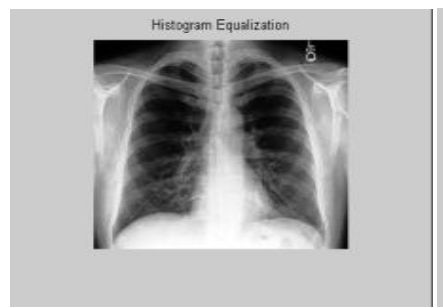

(c)HEQ

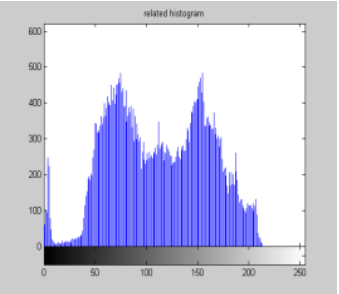

(b) related histogram

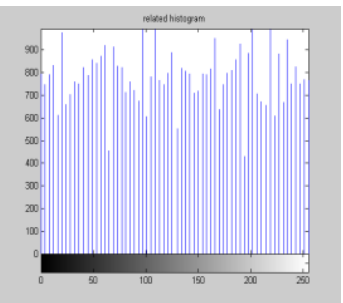

(d) related histogram

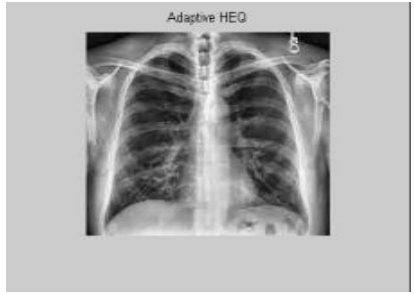

(e)AHE

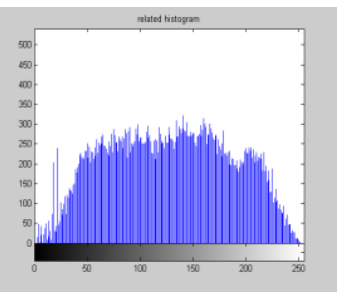

(f) related histogram

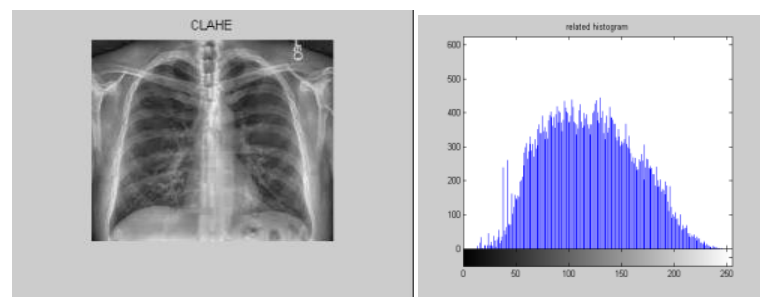

(g)CLAHE (h) related histogram

\begin{tabular}{|l|r|r|r|r|r|}
\hline METHODS & \multicolumn{1}{|l|}{ SNR } & \multicolumn{1}{c|}{ PSNR } & \multicolumn{1}{c|}{ MSE } & \multicolumn{1}{c|}{ AMBE } & ENTROPY \\
\hline HE & 15.134 & 18.164 & 121.12 & 6.243 & 5.761 \\
\hline AHE & 17.212 & 18.298 & 98.10 & 4.134 & 7.312 \\
\hline CLAHE & 19.321 & 20.562 & 57.12 & 1.310 & 2.320 \\
\hline
\end{tabular}

Let us have a discussion about CLAHE how it is superior than $\mathrm{HE}$ and AHE. 
- $\quad$ The HE technique is a global operation hence; it does not preserve the image brightness.

- $\quad$ The AHE Creates some unwanted blurring in edges.

CLAHE, is an improved version of Adaptive Histogram Equalization (AHE).

- $\quad$ CLAHE was originally developed for medical imaging and has proven to be successful for enhancement of low-contrast images such as x-ray images and portal films.

The contrast, especially in homogeneous areas, can be limited to avoid amplifying any noise that might be present in the image.

The only difference between regular AHE and CLAHE is that there is one extra step to clip the histogram before the computation of its CDF as the mapping function is performed.

\section{CONCLUSION}

The three image enhancement techniques such as $\mathrm{HE}$, AHE and ,CLAHE are implemented using MATLAB. The performance of all these image enhancement techniques are analyzed for chest X-ray images are presented. This paper concludes that the CLAHE gives much better results when compare to $\mathrm{HE}$ and $\mathrm{AHE}$.

\section{REFERENCES}

[1] Rafael C. Gonzalez University of Tennessee, Richard E. Woods. Digital image processing Third Edition 2008 .Pearson Education.

[2] Frosio, I. and N.A. Borghese, "Real time enhancement of cephalometric radiographies", Proceedings of the IEEE International Symposium on Biomedical Imaging: Nano to Macro, Apr. 6-9,IEEE Xplore press, Arlington, VA., pp: 972-975.DOI: 10.1109/ISBI.2006.1625082.

[3] Jagatheeswari1, P., S.S. Kumar and M. Rajaram, "Contrast enhancement for medical images based on histogram equalization followed by median filter",Proceedings of the International Conference on Man-Machine Systems. Oct. 11-13, Penang, Malaysia, pp 2A41-2A4 4.

[4] G.N.Sarage, Dr.SagarJambhorkar, ," Enhancement of Chest X-Ray images Using Filtering Techniques", Volume 2, Issue 5, May 2012 ISSN: 2277 128X.

[5] R.B.Tiwari, A.R.YardiDental " $\mathrm{X}$-ray image enhancement based on human visual system and local image statistics", 2012

[6] Ritika, (2012), "A Novel Approach for Local Contrast Enhancement of Medical Images using Mathematical Morphology”, IRACST International Journal of Computer Science and Information Technology \& Security (IJCSITS), ISSN: 2249-9555 Vol. 2, No.2, April 2012.

[7] Kalyan chatterjee, Amit Sur, Prasannjit, Mandavi, Nilotpal Mrinal,"Histogram Based Denoising and Equalization of BioMedical Images" International Journal of Enhanced Research in Science Technology \& Engineering, ISSN: 2319-7463, Vol. 2 Issue 5, May-2013, pp: (57-61).

[8] Siti Arpah Ahmad, Mohd Nasir Taib, Noor Elaiza Abdul Khalid, and Haslina Taib, “An Analysis of Image Enhancement Techniques for Dental X-ray Image Interpretation", International Journal of Machine Learning and Computing, Vol. 2, No. 3, June 2012.

[9] Faisel G.Mohammed, Huda M.Rada, Sega G. Mohammed, 2013, Contrast and Brightness Enhancement for Low Medical X-Ray Images, International Journal of Scientific \& Engineering Research, Volume 4, Issue 5, May-2013. ISSN 2229-5518
[10] Dr. Krishna Mohanta, Dr.V.Khanaa,"An Efficient Contrast Enhancement of Medical X-Ray Images -Adaptive Region Growing Approach", International Journal Of Engineering And Computer Science ISSN:2319-7242 Volume 2 Issue 2 Feb 2013 Page No. 386-390

[11]. Rafael C. Gonzalez University of Tennessee, Richard E Woods, Steven L. Eddins. Digital image processing using MATLAB. Fourth impression 2008 .Pearson Education 